Penultimate draft, to appear in Synthese. The final authenticated version will be available online at: https://doi.org/10.1007/s11229-020-03016-0

\title{
Challenging the Identity Theory of Properties
}

\author{
Vassilis Livanios \\ Department of Classics and Philosophy \\ University of Cyprus \\ vlivan01@ucy.ac.cy
}

\section{Introduction}

According to some philosophers, there is an ontological distinction between dispositionality and categoricality/qualitativity. Property dualists (Ellis 2001; Molnar 2003; McKitrick 2018) think that the distinction, ontologically conceived, grounds irreducible and ineliminable differences between two kinds of natural property which both exist in the world. In contrast to dualists, property monists hold that only one kind of property ${ }^{1}$ actually exists. In particular, categorical monists (Mackie 1977; Armstrong 1997) defend the view that no property (at least no fundamental property) is dispositional, whereas dispositional monists (Mumford 2004; Bird 2007) claim that all properties (at least, all fundamental properties) are dispositional.

In opposition to the previously mentioned authors, a number of metaphysicians hold that the distinction is not ontological and concerns either the predicates we use (Mellor 2000, 767) or the ways we represent and characterise properties (Mumford 1998). The ontological deflationism about the categorical/dispositional distinction may lead to a view called Neutral

\footnotetext{
${ }^{1}$ Not all properties can be meaningfully characterised dispositional or qualitative. The paradigmatic examples of properties that can be so characterised are the natural ones. Hence, in what follows, when I talk about properties, I mean natural properties. Furthermore, since a number of philosophers think that natural relations too (such as the spatiotemporal relations) can be characterised dispositional or qualitative, here the term "properties" is meant to refer to both monadic properties and relations. Finally, as far as I can see, the following discussion is neutral regarding other debates in the metaphysics of properties (for example, the universals/tropes debate).
} 
Monism (NM) about properties. ${ }^{2}$ Neutral monists refrain from offering any ontological account of the nature of properties that would explain the fact that they can be conceived or characterised or described in different ways. In Mumford's words:

...properties themselves are just properties simpliciter, which should be thought of neither as "really" categorical nor "really" dispositional, but which can be denoted in those ways. $(1998,191)$

This paper focuses on another monistic view which has become increasingly popular and whose core tenet is that properties are both dispositional and categorical/qualitative (Carruth 2016; Coates forthcoming; Contessa 2019; Engelhard 2010; Giannotti forthcoming; Heil 2003, 2010, 2012; Jacobs 2011; Jaworski 2016; Martin 1993, 2008; Martin and Heil 1999; Schroer 2010; Strawson 2008; Taylor 2013, 2018). This metaphysical view about the nature of properties could be called Qualitative Powers View, but since some philosophers (including me) use the term "power" to refer to purely dispositional properties, it is better to follow the established terminology and call the view in question Powerful Qualities View (henceforth, $\mathrm{PQV})^{3}$. An important point that needs to be stressed right from the start is that the core claim of PQV, on pain of collapsing to NM, should be construed ontologically: if PQV's core thesis is nothing other than the claim that properties can be conceived or described or characterised both in dispositional and categorical/qualitative ways, then PQV is NM in disguise. If PQV, however, aims to be an ontological middle ground between categorical monism and dispositional monism, it cannot be a form of NM. The interesting fact about PQV is that although it does aim to offer (for the reason given above) an ontological account of the nature of properties (one that is arguably able to explain why properties can be conceived or described

\footnotetext{
${ }^{2}$ I borrow the term from Mumford (1998) who called his own view (according to which properties can be characterised either dispositionally or structurally (categorically) relative to a particular causal role) Neutral Monism.

${ }^{3}$ Given that the term "categorical" is often used in a way that excludes dispositionality, the term "qualities" is not supposed to refer to purely categorical properties. For a discussion about the relation between qualitativity and categoricality in the context of PQV, see Section 3.
} 
or characterised both in dispositional and categorical/qualitative ways), at least one of its versions (the Identity Theory-henceforth IDT) denies that there is an ontological distinction between dispositionality and qualitativity. According to IDT, a property is both dispositional and qualitative because its dispositionality is identical to its qualitativity and both are identical to the property itself (Heil 2003). IDT's denial of an ontological distinction between dispositionality and qualitativity creates an internal tension to the theory to which I shall come back later. PQV is a more general view than IDT, because IDT's identity claim is only one way to interpret the core tenet of PQV. Properties can also be dispositional and qualitative because either they have two distinct parts, one qualitative and one dispositional (Taylor 2018), or are characterised by two distinct (higher-order) properties or aspects (Giannotti forthcoming) 4 .

The aim of this paper is to highlight the difficulties IDT faces regarding three major issues. The first issue concerns the appropriate definition of qualitativity within the context of IDT. Philosophers agree that there is a conceptual distinction between dispositionality and qualitativity. As mentioned previously, however, it is a matter of controversy whether there is also an ontological distinction between them. Although PQV-ists reject that there are two distinct kinds of property (qualitative and dispositional, respectively), they in general allow the possibility of an ontological distinction within the unique (according to PQV) kind of property. Identity theorists, however, hold that the distinction between dispositionality and qualitativity exists only at the conceptual level ${ }^{5}$. The challenge that identity theorists face is to provide a definition of qualitativity which is compatible with the core tenet of their theory and 'support' the distinction between qualitativity and dispositionality.

The second issue concerns the relation between the terms "qualitative" and "categorical". A largely shared opinion is that, though both terms are established in the relevant discussion,

\footnotetext{
${ }^{4}$ For a discussion of the prospects of these alternative versions of PQV, see Livanios (2020).

${ }^{5}$ A perspicuous conception of dispositionality and categoricality would surely help all property theorists to clarify their own metaphysical views on the nature of properties. I would like to thank an anonymous referee for pointing this out.
} 
neither of these terms is free of unwelcome connotations (see Section 3 for some details). Nevertheless, philosophers engaging in the relevant discussion have chosen to use either the term "qualitative" (Carruth, Contessa, Giannotti, Heil, Ingthorsson, Jacobs, Martin) or the term "categorical" (Schroer, Strawson), or both (Taylor 2013). In some cases, the choice between the two terms is presented as a mere terminological matter but as we shall see, given that some of the protagonists of the relevant debate have used the term "categorical" as synonymous to "non-dispositional" (see, for example, Bird (2007)), this is far from true in general. In fact, some versions of PQV (including IDT) should distance themselves from that use of the term "categorical" for reasons of internal consistency.

Finally, the paper includes a comprehensive discussion of what is for most metaphysicians the major issue concerning IDT: that is, the 'surprising' triple identity-claim at the core of IDT. The identity between a property, its qualitativity and its dispositionality certainly needs an interpretation and it is one of the goals of this paper to show that none of the proposed understandings of the identity is capable to 'support' a plausible version of IDT that does not collapse to NM.

The plan of the paper is the following. Section 2 is a thorough discussion of the notion of qualitativity within the context of IDT. In particular, it clarifies the project of identity theorists to provide a definition of qualitativity and explains the basic reasons for the failure of all hitherto suggested definitions. Section 3 is a brief discussion on the relation that qualitativity bears to categoricality and highlights the fact that the proposed by identity theorists metaphysically 'neutral' conceptions of qualitativity threaten to trivialise an important metaphysical debate. Section 4 examines a crucial objection to IDT which concerns the very intelligibility of its core identity-claim. It is argued that none of the extant understandings of the identity in question can adequately meet the intelligibility objection. Finally, Section 5 summarises the challenges to IDT put forward in the paper. 


\section{Understanding qualitativity}

Despite the numerous attempts to define qualitativity, there seems to be no consensus among philosophers on what qualitativity really is. A clear definition of qualitativity would surely promote the metaphysical discussion on the nature of properties, but one might worry that identity theorists have no motivation for seeking a novel definition of qualitativity that is both distinct from the one of dispositionality and compatible with their view. It seems that given a) IDT's core identity-claim, b) an adequate definition of dispositionality and c) Leibniz's law, there is nothing novel to be said about qualitativity. For, given IDT's identity-claim, there can be no features of qualitativity that dispositionality does not possess and, therefore, seeking a definition of qualitativity that differs from the one of dispositionality looks like a doomed project. Are then the attempts of identity theorists to define qualitativity entirely misplaced? I do not think so; surely, according to IDT, qualitativity should share all of its ontological features with dispositionality. Any definition of dispositionality, however, focuses on a subset of those features (the ones been regarded as essential to it). It is possible then that the required (by identity theorists) definition of qualitativity is based on some elements of the set of common features which are not in the 'dispositionality-subset'. To illustrate that, suppose for the sake of exposition that any property $\mathrm{P}$ (at least a fundamental one) is characterised by the following set of ontological features: $\mathrm{SOF}=\{$ simplicity, first-order-ness, independence, intrinsicality, monadicity, actuality, determinateness, ability to confer by itself dispositions on its bearers $\}^{6}$. An identity theorist, in line with her view, should hold that for each property $\mathrm{P}$, its qualitativity Q and its dispositionality D, P and Q and D are all characterised by the totality of the elements of SOF. Nevertheless, she may define qualitativity by appealing to elements of the relative complement of the singleton \{ ability to confer by itself dispositions on its bearers \} with respect

\footnotetext{
${ }^{6}$ All but the last of these features correspond to notions that according to Ingthorsson $(2013,58)$ constitute a proto-idea of what a fundamental property is.
} 
to SOF. (I assume here that the unique member of this singleton is the only definitional characteristic of dispositionality.) Hence, the project of identity theorists is not doomed ${ }^{7}$. Yet, we must be cautious to properly interpret the goal of this project. As already noted, the identityclaim of IDT should be construed ontologically on pain of theory's collapse to NM. In addition, identity theorists need a conceptual distinction between different ways of conceiving or describing a property in order to explain why metaphysicians have come to believe (erroneously in their view) that there could be (or actually exist) two distinct kinds of property. It seems then natural to think that what they want to do by suggesting definitions of qualitativity is to offer an ontological basis for the conceptual distinction between dispositional and qualitative ways of conceiving or describing properties.

Given all that, let me now turn to the proposed (by identity theorists and other metaphysicians) definitions of qualitativity. What I would like to argue in the sequel is that all hitherto suggested definitions are inadequate given the theoretical context of IDT. In what follows, I will present what I think are the basic reasons for the failure of the proposed definitions and classify the latter accordingly.

\subsection{Incompatibility with IDT}

One of the routes actually followed by some metaphysicians is to start with a (relatively uncontroversial) notion of dispositionality and proceed to define qualitativity (or categoricality-for their relation, see Section 3) negatively as non-dispositionality. For instance, Bird claims that:

To say that a property is categorical is to deny that it is necessarily dispositional. (2007, 66-7)

\footnotetext{
${ }^{7}$ It might be objected that identity theorists do not think of the dispositionality and qualitativity in terms of higherorder properties. They surely, however, admit that properties can have features such as first-order-ness, intrinsicality, monadicity, actuality, etc., regardless of how they conceive them.
} 
and Schroer clarifies:

As I am using the term, there is a necessary connection between a dispositional property and the powers it conveys upon its bearer to act in various ways in various circumstances. The essence of a categorical property, in contrast, is not connected to how it makes its bearer behave in various circumstances or causally interact with other objects. $(2013,64)$

To illustrate the problem that emerges from the application of this strategy in the context of IDT, let me now examine a particular case of a 'negative' definition of qualitativity.

\section{The truthmaker account}

Dispositionality is obviously related to the bestowal of dispositions on objects. Yet, this relation to the conferral of dispositions cannot be, without further qualifications, definitional of genuine and irreducible dispositionality. The reason is relatively clear and can be illustrated as follows. First, it is uncontentious that objects have dispositions, at least in the minimal sense that disposition ascriptions of the form "an object $\mathrm{x}$ would do $\mathrm{M}$ under the appropriate circumstances" are true of them (for a discussion on this minimal notion of disposition, see Contessa 2015). Second, there is a consensus among metaphysicians who hold that properties exist as an irreducible ontological category that natural properties are at least partial grounds of the ascription of dispositions. Third, not all philosophers think that natural properties should be genuinely and irreducibly dispositional in order to confer dispositions on objects ${ }^{8}$. Taken all those truths together clearly shows that, unless the best part of the relevant philosophical discussion is misguided, one cannot define (genuine and irreducible) dispositionality via its (alleged) exclusive relation to the bestowal of dispositions on objects. A basic intuition about

\footnotetext{
${ }^{8}$ Contessa (2015) has recently argued that only powers can confer dispositions on their bearers. For a response to his arguments, see Livanios (2018).
} 
dispositionality can, nevertheless, offer a hint regarding the required qualifications: dispositionality can be on its own a source of the modality of the actual world. Dispositions of objects can be fully grounded in properties which either are purely dispositional (that is, they are powers in my terminology) or have dispositional parts or aspects. These properties are inherently modal in the sense that they do not need the 'assistance' of entities of other kinds (such as, par excellence, laws of nature) in order to ground the dispositions that objects possess.

A definition of dispositionality that par excellence respects the above intuition can be offered by the following truthmaker criterion ${ }^{9}$ :

(FOD) The first-order state of affairs of an object instantiating a property that involves dispositionality is by itself (part of) a minimal truthmaker for specific modal truths (often expressed by specific non-trivial counterfactuals) which concern the bestowal of specific dispositions on the object.

Turning now to case of qualitativity/categoricality, the basic intuition about it is that it cannot be by itself a source of the modality of the world. The following truthmaker criterion captures exactly this essential characteristic of qualitativity:

(FOQ) The first order state of affairs of an object instantiating a property that involves qualitativity is not by itself (part of) a minimal truthmaker for specific modal truths (often expressed by specific non-trivial counterfactuals) which concern the bestowal of specific

\footnotetext{
${ }^{9}$ In rough terms, a truthmaker is that in virtue of which something is true. Truthmaker theorists disagree about a lot of issues including what it is to be a truthmaker, what kinds of entities are truthmakers, which truths are eligible to be made true and whether all truths have truthmakers (for a useful discussion, see Macbride 2020). Following the truthmaker criterion commit us only to the minimal claim that a specific range of modal truths (those associated with the bestowal of dispositions on objects) are made true by specific states of affairs.
} 
dispositions on the object. In order to be (part of) a minimal truthmaker for the aforementioned truths, it must be 'supplemented' with appropriate nomic facts.

Assuming that a property may exclusively involve qualitativity, FOQ can easily accommodate the case of purely categorical/qualitative properties. Note however that, on pain of being inapplicable to IDT (and PQV in general), FOQ cannot exclude the case that properties involving qualitativity may involve dispositionality as well. The obvious problem in that case is that since FOQ contradicts FOD (for the latter, in contrast to the former, prescribes that a property is by itself (part of) a minimal truthmaker for specific modal truths which concern the bestowal of specific dispositions on the object), one cannot follow both FOD and FOQ in order to simultaneously ascribe dispositionality and qualitativity to the same property ${ }^{10}$.

A possible way of reconciling the truthmaker-based definitions of dispositionality and qualitativity with IDT is to claim that the definitions in question do not appeal to the same modal truths. More precisely, one might say that a property can be by itself a truthmaker for some modal truths associated with some dispositions of the object possessing it, whereas it needs the 'assistance' of nomic facts in order to be a (partial) truthmaker for other modal truths associated with other dispositions. In fact, this line of thinking constitutes an alternative way of understanding the core thesis of PQV. Properties are both dispositional and qualitative because they have a different truthmaking role (exclusive or partial, respectively) vis-à-vis two different sets of modal truths associated with them. What differentiates, however, the first set of truths from the other? Is it something concerning exclusively the nature of the relevant modal truths or involves in some way the nature of properties as well? An IDT-friendly version of the proposed account should opt for the second option because IDT, in order not to collapse to

\footnotetext{
${ }^{10}$ By presenting this case as an example of the negative-definition-of-qualitativity-strategy, I am not suggesting that identity theorists should opt for a truthmaker criterion both for dispositionality and qualitativity. Nevertheless, it is plausible to think that those identity theorists who wish to have a unified criterion for the categorical/dispositional distinction would at least tend to make such a decision.
} 
$\mathrm{NM}$, should say something about the nature of properties. In the absence, however, of any plausible account of the reasons that would justify the distinction between two kinds of modal truths associated with each particular property, the suggested understanding of IDT seems to be an ad hoc manoeuvre ${ }^{11}$.

To summarise: though a 'negative' definition of categoricality/qualitativity can be embraced by the majority of property theorists, it cannot be endorsed by identity theorists. For, surely, they cannot identify the dispositional with the non-dispositional! Hence, they need to define qualitativity in a clear positive manner which is both adequate and compatible with plausible requirements of their own theory. In what follows I shall show that all notions of qualitativity suggested thus far fail to achieve these desiderata.

\subsection{Limited range of application}

Identity theorists need an account of qualitativity that at least can be applied to all fundamental properties, but some proposed definitions have too narrow scope to fulfil this requirement. Here are two examples of such defective definitions.

\section{Qualitativity as the characteristic of spatiotemporal properties/relations}

Spatiotemporal properties and relations, both fundamental (spatial and temporal location, spatial and temporal orientation, spatial and temporal distance, spatiotemporal invariant interval) and non-fundamental (e.g., shapes of objects) have traditionally been regarded as categorical and therefore, given the arguable identity of qualitative with the categorical (see Section 3), qualitative. Some of those spatiotemporal features are eligible for mathematical descriptions (often provided by the relevant physical theories) which can define them in a

\footnotetext{
${ }^{11}$ An anonymous referee has tentatively suggested that FOD and FOQ can be reconciled with IDT provided that the former refers to modal dispositional truths, whereas the latter to modal qualitative truths. In that case however the pair of the truthmaker definitions would be hardly informative because the distinction between dispositional and qualitative properties would be explained in terms of a distinction between dispositional and qualitative truths.
} 
unique manner and are plausibly considered non-dispositional. For instance, Taylor, in the course of discussing alternative notions of qualitativity, points out that:

...we are able to grasp the property of 'sphericity' by way of a description that does not associate any dispositional features with it, for example: something is spherical iff it is a 3-dimensional object that has all points on its surface equidistant from the centre (in Euclidean geometry). (2018, 1428)

The obvious problem with this account is its limited range of application. (Mathematical) descriptions of spatiotemporal properties, even granted that they are qualitative, obviously apply only to them. Identity theorists cannot ground a general notion of qualitativity in what characterises only a small subset of properties. Notice that the account under consideration does not say that the descriptions of spatiotemporal properties are qualitative qua mathematical but rather due to their 'special nature'. Surely, the account would have a much broader scope in the former case but unfortunately for its potential defenders would also be inadequate. For not all philosophers regard the mathematical descriptions of properties, qua mathematical, as qualitative. Pure powers theorists describe the dispositional role of what they think are fundamental powers (mass, charge, etc.) by mathematical descriptions provided by the relevant physical theories ${ }^{12}$.

\section{Qualitativity as the intrinsic nature of mental qualia}

Mental qualia are supposed to be properties of mental events associated with the phenomenal consciousness, such as the conscious experience of seeing a certain shade of a colour. Some

\footnotetext{
${ }^{12}$ Bartels argues that "Mathematical properties figuring in the representation of fundamental properties are thus not 'only' mathematical properties. The way in which they contribute to the shape of property representations corresponds to the observable physical behavior of the respective properties. ...Since these characteristics, despite of their being not definable by causal roles, contribute to the meaning of physics properties, the meaning of physics properties cannot be exhausted by causal roles." $(2016,92)$. Given that property-representations which are nondefinable by properties' causal roles are most plausibly regarded as qualitative, Bartels in fact argues that some mathematical descriptions of fundamental properties are qualitative. Notice, however, that even in Bartels view, these mathematical descriptions are not qualitative because they are mathematical.
} 
philosophers have proposed that the introspection of mental qualia is in fact an acquaintance with qualitative properties (see, for example, Blackburn $(1990,65)$ and Molnar $(2003,178)$ ). Since the introduction of PQV, the theory has been related to issues of philosophy of mind and especially to the phenomenon of phenomenal consciousness (for two recent examples, see Ingthorsson (2013) and Carruth (2016)) and philosophers such as Jacobs (2011) have suggested that in experiencing a mental quale one has a direct acquaintance with the intrinsic qualitative nature of a powerful quality. Now even granted that the direct introspection of a mental quale offers a qualitative description of a property, the problem with this account (as with the previous one) is that it has a narrow scope; for the qualitativity of most physical properties cannot be described by features of phenomenal experience.

\subsection{Inequivalent descriptions}

It is reasonable to hold that the distinct ways of conceiving or describing properties should be compatible with each other in the context of IDT. The requirement of compatibility can be justified as follows. The ontology of PQV in general allows distinct and incompatible ways of conceiving or describing a property, provided that these ways describe parts or features of the property and not the property in its entirety. It seems, however, that identity theorists in particular need distinct but compatible ways of conceiving or describing a property; for, in order not to collapse their view to NM, they must say something about the nature of a property that can (at least prima facie) explain why we can conceive or describe it in different ways. And it seems difficult to understand how dispositionality and qualitativity, though identical at the ontological level, might explain how we can conceive or describe the same property in incompatible ways ${ }^{13}$.

\footnotetext{
${ }^{13}$ Schroer (2010) and Ingthorsson (2013) argue that the alternative ways of conceiving/describing a property are in fact compatible because they are incomplete and complementary characterisations of it.
} 
But that is not enough; a viable version of the IDT should also need qualitative and dispositional ways of conceiving or describing properties which are on a par. The reason is that any asymmetry between the two kinds of ways (or any priority of one over the other) may create tensions to the theory. For, in that case, identity theorists should offer an account of how dispositionality and qualitativity, though identical at the ontological level, are able to ground descriptions of the same property in inequivalent ways. Just as in the case of incompatible ways we mentioned above, it seems difficult to understand how identity theorists can offer such an account. And once again (and for the same reason as in the case of incompatible ways) they cannot sidestep the difficulty by claiming that the inequivalence of the descriptions is due to epistemic reasons.

Given all that, the problem with some of the proposed definitions of qualitativity is that they do not offer qualitative ways of conceiving or describing properties which are on an equal footing to the dispositional ones. The inequivalence of the descriptions consists in the fact that only the dispositional ways can also provide (under certain assumptions) identifications of the properties. To illustrate that, let us start with what is unanimously regarded as a dispositional way of conceiving/describing a property. Everyone agrees that in order to describe/conceive a property dispositionally you should appeal to its causal/nomic role. In rough terms ${ }^{14}$, this role is exhausted by the totality of causal/nomic relations the property bears to other properties and is exposed by describing what objects would do in various circumstances in virtue of possessing that property. The crucial point is that the causal/nomic role of a property can be (and actually often is) used to uniquely identify it. Let us now turn to the qualitative/categorical way of describing/conceiving a property. In order to be on a par with the dispositional way, it should be capable of offering an identification of a property. The following examples show that some proposed definitions fail to accomplish that task.

\footnotetext{
${ }^{14}$ Hawthorne (2001) provides a precise definition of the causal/nomic role of a property in more formal terms.
} 


\section{Identifying qualitativity with actuality}

In his (2012), Heil claims:

At any rate, qualities are categorical; qualities are here and now, actual, not merely potential, features of the objects of which they are qualities. $(2012,59)$ (emphasis added)

Strawson also claims that:

...all actual concretely existing being is ipso facto categorical being ${ }^{15} \cdot(2008,278)$

Both quotes suggest that qualitativity is intimately related to actuality. Can an identity theorist identify the former with the latter? In a sense, she can; given IDT's triple identity-claim and the fact that the vast majority of contemporary metaphysicians (both property monists and those who think that there exist two kinds of properties) agree that all properties are actual, an identity theorist can identify actuality with both qualitativity and dispositionality. In that case, however, she cannot ground (on the basis of the proposed identification) the required for IDT conceptual distinction between qualitativity and dispositionality. Even if identity theorists identify qualitativity with actuality, the resulting qualitative description of any property, though obviously compatible with its dispositional description, cannot be on a par with it. The problem, of course, is that actuality cannot discern one property from the others since all natural properties are actual. Consider, for example, the dispositional way of describing positive electric charge. Charged objects repel or attract other charged objects, generate currents, produce electromagnetic fields around them, etc. Charge has a nomic role, described by the totality of nomic relations that bears to other properties, by which we can identify it as a natural property. Consider now the qualitative way of describing charge: charge is an actual property. But so is mass, spin, etc. The two ways of describing charge are certainly not on a par.

\footnotetext{
15 Strawson uses the term "categorical" instead of the term "qualitative".
} 
Identifying qualitativity with 'something' that makes space occupants differ from empty space Schroer (2013) defines qualitativity as a primitive 'something' that all substantial space occupants share and makes them different from empty space. Schroer's 'thin' conception of qualitativity can ground a conceptual distinction between it and dispositionality. Furthermore, provided that all natural properties are space occupants (of course not all philosophers agree on that), this qualitative description is compatible with any dispositional one. The difficulty once again is that the qualitative description is not on a par with the dispositional one since the former, in contrast to the latter, cannot differentiate between distinct properties.

Qualitativity as the intrinsic nature of physical qualia (Jacobs' thick quiddities account) Jacobs (2011) proposes that we should identify qualitativity with thick quiddity. But what is a thick quiddity? To illustrate the meaning of this notion we should first disambiguate the meaning of quiddity itself. According to one interpretation, quiddities of properties are distinct (though non-separable) from properties themselves. The term "quiddity" refers to an intrinsic feature of a property, a second-order property or aspect. A definition of a quiddity in this sense is: $\mathrm{Q}$ is a quiddity $=(\mathrm{P})(\mathrm{Q}$ is the property of being identical with $\mathrm{P})$, where $\mathrm{P}$ is a first-order property. Jacobs, however, appeals to another sense of quiddity. According to this alternative sense, quiddities are just first-order properties that describe the essential natures of concrete individuals. A quiddity of a property is nothing other than the property itself. A prominent philosopher who has identified quiddities with first-order properties (albeit only the categorical ones) is David Armstrong. In his (1997), Armstrong identifies natural categorical properties with thin quiddities which, within each adicity class of properties, differ merely numerically. In his earlier work (1989), however, Armstrong had endorsed a different, thick sense of quiddities which differ from each other not merely numerically, but by their nature. Jacobs' quiddities are just Armstrong's thick quiddities. 
The problem with Jacobs' proposal is that the notion of thick quiddity cannot ground a qualitative way of describing a property that is on a par with its dispositional way. To see that, consider Armstrong's $(1997,168-9)$ reason to reject thick quiddities: thick quiddities involve ontological 'embarrassment' since science does not seem to concern itself with them. Positing thick quiddities we just say that there is 'something' at the ontological level, a nature, that could identify a property (recall that thick quiddities, in contrast to thin ones, do differ by their nature). This however hardly provides a qualitative description of the property which is clearly distinct and on a par with the dispositional one. Furthermore, given the indifference of science to quiddities, it is hard to think that there could be a qualitative scientific (perhaps mathematical) description which might be plausibly grounded in thick quiddities.

The objection from the inequivalence of the two ways of describing/conceiving properties applies also to the following two definitions of qualitativity. The first is suggested by Giannotti (forthcoming):

...at least some properties of our world appear to be both dispositional and qualitative in character. Consider mass and charge, two putative fundamental properties. In virtue of having a certain mass, a particle is disposed to generate a gravitational force. But having a certain mass is also a qualitative feature of a particle. By having a certain mass, a particle has a certain quantity of matter. (forthcoming, 3 , emphasis added) ${ }^{16}$

To illustrate Giannotti's proposal, consider a determinate of ten micro-coulombs of electric charge. According to Giannotti, a qualitative way of describing this determinate is simply: "a property of ten-micro coulombs". One might think that such a description is on a par with the dispositional one because it seems that, by saying that a certain object possesses ten microcoulombs of charge, we actually identify the property that that object instantiates. This, however, presupposes that we have already identified coulombs as units of electric charge. As

\footnotetext{
${ }^{16}$ Since Giannotti's paper is forthcoming, the reference is to page number of the online-first version of the paper.
} 
a result, the expression "a property of ten-micro coulombs" is in fact tantamount to the expression "a property that possesses a certain quantity of amount of 'charge-stuff"”. Since, however, the problem is how to identify the 'charge-stuff', the proposed qualitative description is hardly identificatory (and, as a result, not on a par with the dispositional way of describing charge).

One might try an alternative, modal definition of qualitativity and claim that qualitative is what has a primitive interworld identity. Yet, this proposal has two features that make it inappropriate for the purposes of IDT theorists. First, given that the dispositional way of description via causal/nomic roles is often taken as a way of interworld identification of properties, the proposed qualitative way seems to be incompatible with the dispositional one (for, according to this proposal, identity theorists should claim that properties both have and not have a primitive interworld identity). Second, it cannot offer, as it stands, an identificatory description which is on a par with the dispositional one.

\subsection{One last account}

I would like to conclude the discussion on qualitativity by examining one last account.

\section{Qualitativity as invariance under the action of symmetry transformations}

Contemporary physics suggests that fundamental properties can be described or conceived or identified as invariants under the action of fundamental physical symmetries. For instance, rest mass and spin of elementary particles can be identified as distinct invariants under the action of the so-called Poincare group of transformations which is associated with the symmetry under the action of Lorentz boosts and rotations, and of space-time translations (for details, see Livanios (2017)). Although I know of no identity theorist who has suggested that, a plausible proposal is that the aforementioned description, qua mathematical description analogous in a 
sense to the definition of sphericity previously mentioned, is a qualitative one. The symmetrybased description, as physics clearly shows, is compatible with the causal-role-based dispositional description and is distinct from it. It is distinct from the dispositional description because the latter is given in terms of causal laws that properties 'obey', whereas the symmetrybased description focuses on the invariant character of properties under the action of specific fundamental symmetries. And it is compatible with it because physicists use both descriptions without contradiction. Furthermore, it seems that the symmetry-based description is on a par with the dispositional one since, by definition, it provides an identification of properties. Is then this invariance-description what identity theorists look for? I think not, mainly for the following reason. It is not enough to claim that symmetry-based descriptions are qualitative. We have to clarify why they are qualitative. Is it merely because they are mathematical? Or the qualitative character has something to do with the invariance itself? We have to reject the former option. As mentioned previously, dispositional descriptions can also be expressed in mathematical terms, at least in the case of fundamental properties (that is, they are descriptions which consist in a set of mathematical identities that express law statements associated with the properties in question). Hence, it is not due to its mathematical character that the invariancedescription is considered qualitative. So, it must be the invariance itself that guarantees the qualitativity of the description. There is a problem, however, with that suggestion. In contrast to the dispositional description which is based on the main intuition about dispositionality (that is, the intuition that dispositionality by itself bestows specific dispositions on objects) and is able to offer an identification of properties (via their causal/nomic roles), the 'qualitative' symmetry-based description can only play the latter role without simultaneously having any obvious relation to any notion that could be called qualitative. Let me make this point clearer. Symmetries in general express situations of equivalence between various elements. The presence of these equivalent elements is most often scientifically understood in terms of the 
irrelevance of certain properties to physical descriptions. For instance, the equivalence of spatial locations (due to the symmetry of spatial translation) shows the irrelevance of the property of absolute spatial position to physical descriptions. For the physicist, the above remarks indicate that properties such as the absolute spatial position are eliminable precisely because they are physically irrelevant. A scientifically informed metaphysician might follow the physicist at this point and argue that properties defined as the changeable elements under symmetry operations admitted by mature and successful physical theories do not exist. Invariance under the action of symmetry transformations can be connected then only with the real existence of properties and has no obvious links to either qualitativity or dispositionality. Given that, an invariance-based description of a property can (at best) be construed as qualitative only negatively; that is, as a non-dispositional description. This choice is available for most property theorists but, as I have already explained, identity theorists cannot think qualitativity as non-dispositionality on pain of contradiction. They have, therefore, no cogent reasons to identify qualitativity with invariance under the action of symmetry transformations ${ }^{17,} 18$.

In a nutshell: since identity theorists insist that properties are both dispositional and qualitative, they need to define qualitativity in a clear positive manner which is both adequate and compatible with plausible requirements of their own theory. Although I have not proved

\footnotetext{
17 An anonymous referee has suggested that, given symmetries' connection (via Noether's theorem) with conservation laws, there is something odd in using symmetry transformation invariance as a way to define qualitativity. The main reason that supports referee's claim is that conservation laws describe (a part of) the causalnomic role of properties and as a result the symmetry-based definition is in fact associated with dispositionality. This could be an extra reason to reject the symmetry-based definition of qualitativity. Yet, given that the latter is supported by the purely conceptual way of finding the invariants of certain transformations via the group structure that emerges from their repeated application, it is not clear to me that the symmetry-based definition is intimately related to properties' causal roles and hence to dispositionality. The case would be different if one can convincingly argue that symmetry principles are in a sense equivalent to conservation laws. Yet, as argued in Livanios (2010), there are strong reasons to reject such an equivalence.

${ }^{18}$ It might also be pointed out that the symmetry-based description cannot offer a broad enough notion of qualitativity since it is applicable only to fundamental properties. This objection, however, cannot move those metaphysicians who think that the debate about the proper account of the nature of properties should be confined to the fundamental level.
} 
that they cannot provide such a definition, the failure of all extant proposals clearly suggests that the prospects of providing the required account are slim. In any case, as long as identity theorists are not offering it, there is something problematic at the very foundations of IDT.

\section{Qualitativity and categoricality}

The relation categoricality bears to qualitativity is another confusing aspect of the discussion on IDT (and PQV in general). Martin and Heil, the main proponents of IDT, tend to use the term "qualitative" instead of the term "categorical":

... the dispositional is as real and irreducible as the categorical. (Or, as Martin would prefer to say, the dispositional is as real and irreducible as the qualitative. Talking of the distinction as being between the dispositional and the categorical can suggest that dispositionality is not really categorical: not really 'there' in the object.) (Martin 1996, 74)

...talk of categorical properties might usefully be understood as an oblique, arguably misleading, way of talking about qualities. At any rate, qualities are categorical; (Heil 2012, 59)

Most possibly, their motivation is to avoid the unwanted connotations of the term "categorical" which, in the context of the categoricalism/dispositionalism debate, is often defined in opposition to the term "dispositional". Of course, the term "qualitative" is not free from irrelevant (to the debate under consideration) connotations. In some cases qualitative features are used in contrast to relational ones, while in others they are contrasted with quantities. Furthermore, a well-known philosophical distinction is between qualitative and non-qualitative properties (see Cowling 2015), where paradigmatic examples of the latter are haecceities (properties associated with the identity of specific individuals) and impure properties (features that have various 'ties' to specific individuals). Given all that, one might claim that the term 
"qualitative" has (due to the numerous alternative meanings) more unpleasant connotations than the term "categorical" and, in that sense, it may more easily lead someone who uses it astray. Be that as it may, the real problem with preferring the term "qualitativity" over the term "categoricality" lies elsewhere. As presented in the previous section, most of the definitions of qualitativity are deliberately metaphysically 'neutral' (as far as the relevant debate is concerned) to avoid any internal contradiction of IDT. They are related to features that both categoricalists and pure powers theorists can accept. As Taylor (2018) points out:

Though pure powers theorists do not mean to deny that properties are 'qualities' in the senses in which the identity theorists claim that properties are qualities, it is certainly true that pure powers theorists deny that properties are 'categorical'... When pure powers theorists deny that properties are categorical, they mean the Armstrongian sense of 'categorical'... properties that are defined in opposition to dispositions/powers. $(2018,1432-3)$

This neutrality, however, is problematic; for it is supposed that IDT is a metaphysically interesting middle path between the 'extremes' of pure categoricalism and pure powerism as they are traditionally conceived. Identity theorists, however, have changed the rules of the game. They actually have 'absorbed' any metaphysically robust notion of categoricality into their notion of powerful qualities and made all properties almost trivially categorical. (This is clear, for instance, in the case of Strawson (2008) who identifies categoricality with being in general.) By doing that, identity theorists have actually trivialised the traditional debate rather than proposing a metaphysically interesting middle view between the 'problematic' extreme views. It might be objected that there never was a metaphysically interesting distinction to debate about after all and IDT has revealed that. I disagree; the debate concerning the categorical vs dispositional nature of properties, as traditionally conceived, actually concerns the choice of a general worldview. It is about the question whether properties by themselves 
confer dispositions on their bearers (and so they are inherently modal and active) or need the 'assistance' of laws of nature to do that. This is by no means a trivial or uninteresting metaphysical issue.

The failure of IDT (as has been actually developed) to be a metaphysically interesting middle view in the context of the categoricalism/dispositionalism debate does not rule out the possibility of an alternative understanding according to which PQV in general has (at least prima facie) a metaphysically interesting territory to cover. To see that, recall that the traditional debate has thus far been an all-or-nothing affair. Properties either need laws to make them powerful (qua categorical properties) or not (qua powers). A tentative suggestion is to understand PQV as a middle path between pure categoricalism and pure powerism in the sense that a property can bestow dispositions on its bearers partly because of its nature and partly because of the laws of nature. For instance, one might claim that mass bestows massive objects the disposition to attract other massive objects but Newton's gravitational law is also needed to determine the exact strength of that attraction. Or, that mass has an 'ultra-thin' power to be nomically governable and Newton's gravitational law determines both the exact strength and the attractive character of the force between two massive objects. According to such a position, both properties and laws are modality sources in the actual world ${ }^{19}$. Understanding PQV in such a manner is prima facie promising but it is beyond the scope of this paper to discuss the prospects of this view. So, let me now proceed to another major issue concerning IDT, the 'surprising' triple identity between a property, its dispositionality and its qualitativity/categoricality.

\footnotetext{
${ }^{19}$ For details of such a dualist account, see Ioannidis, Livanios and Psillos (2021).
} 


\section{The 'surprising' triple identity}

Martin (1997, 216; see also 2008, 65-6)) was the first to introduce the triple identity which is the core tenet of IDT:

For any property that is intrinsic and irreducible, what is qualitative and what is dispositional are one and the same property viewed as what that property exhibits of its nature and what that property is directive and selective for as its manifestations. These cannot be prised apart into the purely qualitative and the purely dispositional. What is exhibited in the qualitative informs and determines what is the forness of the dispositional, and what is the forness of the dispositional informs and determines what is exhibited in the qualitative. There is no direction of priority or dependence. There is no reduction of one to the other. The only way that this can be expressed is by claiming that the qualitative and dispositional are identical with one another and with the unitary intrinsic property itself. This is perhaps a surprising identity, but frequently it happens that different representations turn out to one's surprise to be of the identical entity. (emphasis added) $)^{20}$

Here is Heil $(2003,111)$ expressing the surprising identity of IDT in more formal terms:

If $\mathrm{P}$ is an intrinsic property of a concrete object, $\mathrm{P}$ is simultaneously dispositional and qualitative; P's dispositionality and qualitativity are not aspects or properties of P; P's dispositionality, $\mathrm{P}_{\mathrm{d}}$, is $\mathrm{P}$ 's qualitativity, $\mathrm{P}_{\mathrm{q}}$, and each of these is $\mathrm{P}: \mathrm{P}_{\mathrm{d}}=\mathrm{P}_{\mathrm{q}}=\mathrm{P}$.

The most discussed objection against IDT questions the very intelligibility of the triple identity-claim (Coates forthcoming; Contessa 2019; Giannotti forthcoming; Ingthorsson 2013; Jacobs 2011; Martin 1997; Schroer 2013; Strawson 2008; Taylor 2013, 2018). Let us call this

\footnotetext{
${ }^{20}$ According to Martin's (1993) earlier view (which he called the Limit View), qualitativity and dispositionality are distinct in abstraction but ontologically inseparable: "On this understanding of the view, no property is purely dispositional or purely qualitative. Properties are purely dispositional or purely qualitative 'only at the limit of an unrealizable abstraction' and, thus, the qualitative and the dispositional natures are 'abstractly distinct but actually inseparable" $(1993,184)$. In his mature view (that is, IDT) the ontological inseparability of qualitativity and dispositionality is explained by the fact that they are identical.
} 
objection the intelligibility objection. We may discern an ontological and a conceptual/descriptive version of the intelligibility objection. According to the former:

(OIO) It is absurd to identify qualitativity and dispositionality because they are ontologically incompatible.

Identity theorists may respond to OIO by claiming that it begs the question against their view. For them there are no cogent arguments for the thesis that qualitativity is ontologically incompatible with dispositionality. In fact, they hold the stronger view that the ontological compatibility of qualitativity and dispositionality is guaranteed by their identity which in turn might be justified as the best explanation of their (alleged) inseparability. So, for them, it is not at all absurd to identify qualitativity and dispositionality at the ontological level.

I am not going to challenge here the above response though I think that it is difficult to understand the metaphysical significance of the categorical/dispositional debate if qualitativity and dispositionality are not ontologically distinct (recall the previous remarks on the different worldviews in Section 3). To introduce the second version of the intelligibility objection let me first remind the reader that according to IDT's basic claim, for each property P, P's qualitativity is identical to P and P's dispositionality is also identical to P. Hence, when someone claims that a property is qualitative, she ipso facto says something that exhausts the nature of the property. Similarly, when she claims that a property is dispositional, she (again) ipso facto says something that exhausts the nature of the property. In the context of IDT, therefore, it is misleading to say that qualitativity or dispositionality does not exhaust the nature of a property (see also Taylor 2018, 1434). Yet, the fact is that according to IDT neither the qualitative nor the dispositional description/conception exhausts by itself the ways we can describe or conceive a property. Given a) the indispensability of both ways for the exhaustive description 
of a property, b) the identity between qualitativity and dispositionality and c) the requirement that something at the ontological level must explain somehow our ability to describe or conceive properties in alternative ways (recall that IDT should offer such an explanation in order not to collapse to NM), the conceptual/descriptive version of the intelligibility objection can be (in rough terms) expressed as follows:

(DIO) There is no plausible explanation of the fact that qualitativity and dispositionality, though identical at the ontological level, can 'support' distinct (and perhaps incompatible) ways of describing/conceiving a property.

The most popular strategy to deal with DIO appeals to the notion of partial consideration. Martin introduced that strategy (and Heil followed him) in order to show how one can coherently hold that qualitativity is identical to dispositionality despite the fact that they appear different:

What is qualitative and what is dispositional for any property is less like a two-sided coin or a Janus-faced figure than it is like an ambiguous drawing. A particular drawing, remaining unitary and unchanged, may be seen and considered one way as a gobletdrawing and differently considered, it is a two-faces-staring-at-one-another-drawing. The goblet and the faces are not distinguishable parts or components or even aspects of the drawing, although we can easily consider the one without considering, or even knowing of, the other. The goblet-drawing is identical with the two-faces drawing. (Martin 1997, 216-217)

the inseparability of a property's dispositionality and qualitativity is analogous to the inseparability of the old lady and the young woman in Leeper's famous ambiguous figure... (Martin and Heil 1999, 46-47) 
A property's dispositionality and its qualitativity are, as Locke might have put it, the selfsame property differently considered. (Heil 2003, 112)

My reading of the task of the partial consideration strategy is to provide a response to the conceptual version of the intelligibility objection in the following sense: the strategy aims to offer an account of how qualitativity and dispositionality, though in fact identical, can be regarded as 'distinct' ontological elements that 'support' distinct ways of conceiving/describing a property. This interpretation of the goal of the partial consideration strategy may seem odd given identity theorists' explicit denial of any ontological distinction between qualitativity and dispositionality. Yet, it is in line with the major requirement the fulfilment of which can distinguish IDT from NM: the identity theorist should say something at the ontological level to explain why we have distinct ways of conceiving/describing a property. Saying that, I am not committed to the view that identity theorists have actually succeeded in this task. In fact, my purpose is to show that they have hitherto not succeeded. To this end, in what follows, I present the alternative ways of understanding the triple identity of IDT which, in my interpretation, correspond to different ways of implementation of the partial consideration strategy to meet DIO.

By definition, to partially consider an entity is to attend to it under one concept while ignoring the possibility of considering it under an alternative concept. Implementing the partial consideration strategy to the case of properties, we can then say that to partially consider a natural property as either dispositional or qualitative is to attend to the property under one concept (let us say, the one corresponding to dispositionality) while ignoring the possibility of considering it under the alternative concept (the one corresponding to qualitativity). Martin and Heil suggest that the way we understand how we can consider in different ways ambiguous figures may shed light, qua analogous, on the case of the identity between dispositionality and qualitativity. The analogy, however, fails due to the following asymmetry: while one cannot 
simultaneously see a figure as a duck and a rabbit, he can simultaneously consider a property as dispositional and qualitative. Of course, at each instant of time, one person can see the ambiguous drawing as a duck and another person as a rabbit, but the fact remains that the same person cannot do simultaneously both. An important consequence of this asymmetry is that only in the property case one might claim that the alternative descriptions are in fact incomplete complementary characterisations of the same property (for a defence of this view about the alternative descriptions, see Schroer 2010 and Ingthorsson 2013). One cannot say something similar for the ambiguous drawings. Seeing a specific drawing as a duck (rather than a rabbit) is not an incomplete description of it. Seeing the same drawing as a rabbit is not required in order to have a complete description of the drawing. Given all that, the property-ambiguous figure analogy breaks down.

One might try to restore Martin-Heil's analogy by claiming that we cannot simultaneously conceive a property as qualitative and dispositional. A necessary (but not sufficient) condition for that claim to be true is to hold that the qualitative conception is a non-dispositional conception. For instance, if we think that the dispositional conception of a property presupposes the essential directedness for specific manifestations of behaviour, whereas the qualitative one has only a contingent association with those manifestations, then an identity theorist cannot simultaneously conceive a property in qualitative and dispositional ways on pain of contradiction. But for identity theorists even the necessary condition does not always hold because, in their view, we can have qualitative conceptions of a property which are compatible with the dispositional ones (see Section 2).

It might be objected that, despite the above-mentioned disanalogy, the duck-rabbit example can illuminate the triple identity-claim of IDT provided that the aspect which is not analogous in the two cases is inessential for understanding the core tenet of IDT. Yet, this is not the case because the disanalogy is related to an element the appeal to which is (at least according to 
some PQV-ists) crucial for the understanding of IDT's identity claim: it is only in the property case that the alternative descriptions can be considered as incomplete complementary characterisations of the same entity. (I would like to thank an anonymous referee for raising a closely related worry.)

Another philosopher who suggests that we can understand the 'surprising' identity via an analogy is Jonathan Jacobs (2011). He proposes that IDT's identity is analogous to a Kripkean a posteriori identity. Jacobs appeals to the truthmaking relation in order to show how we can imagine that qualitativity and dispositionality might have been distinct, though they are in fact identical. For Jacobs:

To be qualitative is to be identical with a thick quiddity (a quality or quale), as discussed below. ${ }^{21}$ To be powerful is to be a nature sufficient to be (part of) the truthmaker for the counterfactuals describing what objects with that property would do in the various circumstances they might find themselves in. (If truth bearers are necessary existents, then to be powerful is to be (part of) the truthmakers for such counterfactuals.) The qualitative is identical with the powerful; one and the same thing is both identical with a thick quiddity and a nature sufficient to be (part of) the truthmaker for the counterfactuals. $(2011,90)$

Jacobs does not offer an explanation why a specific thick quiddity is also sufficient to make true certain counterfactuals. Since, however, this brute fact is 'forced' on us by the a posteriori surprising discovery of the identity between dispositionality and qualitativity, he tries to distinguish their conceptions in order to make us capable of imagining them as distinct, though they are actually identical. Assuming that Jacobs provide a plausible account of the conceivability of distinctness between dispositionality and qualitativity, their actual identity can be explained in IDT-friendly terms in two different ways: first, an identity theorist may

\footnotetext{
${ }^{21}$ For the notion of thick quiddity that Jacobs invokes, see Section 2.
} 
hold that the conceivability in question does not imply the metaphysical possibility of a scenario in which dispositionality and qualitativity are distinct. If the distinctness-scenario is not even metaphysically possible, we have no cogent reason to reject IDT's identity-claim. Second, an identity theorist may admit that conceivability entails metaphysical possibility (at least in this case) but claim that what is possible is not a scenario in which dispositionality and qualitativity are distinct. It is rather a scenario in which dispositionality ${ }^{*}$ and qualitativity ${ }^{*}$ are distinct, where dispositionality* $\neq$ dispositionality and qualitativity* $\neq$ qualitativity. Again, if that is true, there is no convincing reason to reject IDT's identity claim.

Nevertheless, we might reasonably ask: what are the a posteriori grounds for the triple identification of the property with dispositionality and qualitativity? Surely, identity theorists can (and actually do) provide examples from the realm of non-fundamental properties of macroscopic objects to convince us that each property has simultaneously a categorical and a dispositional 'nature'. The oft-cited example is a ball which, due to its spherical shape, can roll down an inclined plane. This example seems to show that paradigmatic qualitative properties such as the geometrical properties of macroscopic objects confer (by themselves?) causal powers on their bearers and, so, are also dispositional. Let us grant that such examples provide a posteriori evidence that properties play a truthmaking role regarding both 'qualitative' and 'dispositional' truths. It is something about the property of sphericity that makes true the 'qualitative' truth that all surface points of the ball are equidistant from its centre and something about the sphericity again that makes true the 'dispositional' truth that the ball can roll down an inclined plane. Is there, however, any a posteriori evidence that might rule out the possibility that each property has two distinct but non-separable 'aspects', one of which makes true the 'qualitative' truths and the other the 'dispositional' truths? If there is not (as I think it is the case), what reason do we have to identify these 'aspects' with the property itself? As far as I 
can see, Jacobs does not provide one ${ }^{22}$. Though his theory provides a promising response to the conceptual version of the intelligibility objection, it is not clear whether it also provides an adequate explanation of IDT's triple identity.

In contrast to Jacobs who, like Martin and Heil, defends the plausibility of his version of IDT by appealing to an analogy, Schroer aims to support IDT's identity claim not via analogies but by defining the qualitativity in such a way so as to make its separability from dispositionality less plausible:

... this new conception of categoricity should make a difference with regard to the intuitive credibility of the Partial Consideration Strategy in the context of the dispositional/categorical properties case. Given this new conception of categoricity, there would not be the same prima facie case for thinking that both dispositional properties and categorical properties are stand-alone entities, for our grasp of categoricity would no longer reveal it to have a rich nature in its own right. (In this manner, our grasp of the categoricity becomes more like our grasp of the substratum.) There would also not be the same prima facie case, involving various conceivability arguments, for thinking that dispositionality and categoricity are metaphysically separable; unlike the $\mathrm{M} / \mathrm{H} / \mathrm{S}$ position, this new account would not entail that different types of categoricity are metaphysically inseparable from different types of

\footnotetext{
${ }^{22}$ According to one interpretation, Tugby's (2012) Qualitative Dispositional Essentialism (QDE) is a view very close to Jacobs' version of IDT. For Tugby, properties have intrinsic qualitative natures which essentially bestow certain dispositions on their bearers. If these natures exhaust the nature of the properties (as the following quote suggests) then QDE is intimately related to Jacobs' identity theory:

“... we could understand the claim that quality instances essentially dispose their possessors towards certain behaviour in the following way: a thing's property instantiations entirely constitute the truthmakers for certain counterfactuals true of that thing. Schematically, we may put this as follows: QDE schema: where P is any natural property, necessarily, if $x$ has $P$, then in virtue of $x$ 's being $P$, if $x$ were $F$, then $x$ would be G (ceteris paribus)." $(2012,728)$
}

Nevertheless, other passages suggest that properties have qualitative aspects and, so, QDE is not a version of IDT:

"It is, instead, purely the qualitative aspects of a thing's properties which ground its dispositions." (ibid.) 
dispositionalities, for, according to this new conception, there are not different types of categoricity. $(2013,76)$

As remarked in Section 2, Schroer defines qualitativity as a primitive 'something' that makes space occupants differ from empty space and claims that different properties all contribute the same determinate qualitativity (categoricity, as he calls it) to their bearers. In Schroer's view, then, each property is both dispositional and qualitative in the sense that it is an ontologically simple entity that makes its bearers differ from empty space and essentially bestows specific dispositions on them. As Taylor (2013), however, argues, Schroer's unique determinate qualitativity combined with IDT's identity-claim has the unpalatable consequence that all determinate properties, regardless of the determinable they fall under, are identical to this unique qualitativity and, so, are themselves identical. Even if one interprets Schroer's view as allowing a diversity of determinate qualitativities of a single determinable qualitativity which all kinds of properties 'share', the consequences are still problematic. For in that case, there is only one kind of determinable property in the world; it is the one which is identical to this unique determinable qualitativity.

Another philosopher who attempts to make IDT's identity claim plausible (or at least intelligible) by appropriately defining qualitativity (categoricality in his terms) is Galen Strawson (2008). As remarked in Section 2, for Strawson all actual concretely existing being is categorical being. His point then is that dispositional being, like all being, is categorical as well. In particular, Strawson's argument for the 'surprising' identity of the categorical and the dispositional is the following:

a. Nothing can possibly have the (total) categorical being that it has and not have the (total) dispositional being that it has and vice versa. $(2008,276)$ 
b. Therefore, there is no real distinction between an object's total categorical being (properties) and its total dispositional being (properties).

c. If there is no real distinction between those beings then they are identical.

Premise (a) is a claim for the necessary inseparability in reality of the dispositionality and the qualitativity. Even if we agree with Strawson about the truth of (a), the argument does not run because the move from (a) to (b) and (c) (that is, from the necessary inseparability of the dispositional and the categorical to the lack of a real distinction between them and to their identity) is illegitimate. The separability of two entities is only a sufficient but not a necessary condition for there to be a real distinction between them. It might be objected that if, pace Strawson, there exists a real distinction between dispositionality and qualitativity, we have no cogent explanation of their inseparability. Oderberg $(2009,678)$ proposes as a response that in all cases where we have real distinctions between inseparable entities it is the essences of the latter that best explain their inseparability. Irrespective of the plausibility of Oderberg's view, what matters for the present discussion is that Strawson's argument does not seem to provide a cogent explanation of the 'surprising' identity-claim of IDT.

Finally, Engelhard (2010) offers an alternative interpretation of what an identity theorist does when she partially considers a property as dispositional or qualitative. Engelhard gets her inspiration from Jonathan Lowe's 4-category ontology and his view about the dispositional/occurrent distinction. In his (2006), Lowe introduced a contrast between occurrent and dispositional states of affairs. According to his definition, an occurrent state consists in the possession of some mode by a particular object, whereas a dispositional state consists in some particular object's instantiating a kind-universal which, in turn, is characterised by some property-universal (ibid., 134). For Lowe, though the dispositional/categorical distinction (interpreted as dispositional/occurrent distinction) has 
nothing to do with types of property but rather with two distinct types of indirect characterisation of objects by properties, it has nonetheless an ontological ground. The latter is provided by an ontology of four categories (particular objects, modes, substantial kinduniversals and non-substantial property-universals) interrelated via distinct formal relations. According to Lowe's 'ontological square', objects and modes instantiate kinds and propertyuniversals respectively, while kinds and objects are characterised by property-universals and modes respectively.

Engelhard's proposal gets rid of natural kinds and focuses on the fact or state of affairs of an object instantiating a property. For her, when we consider a property as dispositional, we consider it in abstraction from the fact or state of affairs which it is part of (that is, we consider it as a universal). Engelhard claims that by abstracting away the particular which is the other constituent of the state of affairs "we can detect the nomic relations that the property involves that are the expression of its modal character" $(2010,53)$. In contrast to the dispositional way of conceiving, Engelhard argues, when we consider a property as qualitative we do not abstract from the fact which it is part of; rather we consider the property as a mode of a particular, what makes a specific particular be a specific way. The problem with Engelhard's view is that a qualitative characterisation of a property does not presuppose a description of what makes a particular object be a specific way. One might have a qualitative description of a property by describing what makes similar (vis-à-vis the property in question) objects be a specific way. In that case, however, we need (as with the dispositional description) to abstract from particular facts or states of affairs. It is not clear, therefore, whether the distinction between dispositional and qualitative ways of considering properties has anything to do with the contrast between considering a property as universal and considering it as a mode of an object.

I have thus far examined the different ways of understanding IDT's triple identity and argued that all of them face difficulties. Now that we have a more comprehensive view it is 
time to present a general problem that besets any account of the triple identity that is supposed to meet DIO by invoking the partial consideration strategy. Let me begin by stating the obvious fact that the partial consideration strategy is based upon a mental activity. We have in front of us an instance of a property and, somehow, we consider it either as dispositional (while ignoring the alternative qualitative conception) or as qualitative (while ignoring the dispositional conception). As I have repeatedly pointed out in this paper, IDT is not supposed to be NM in disguise. A neutral monistic approach may rest content with a purely epistemic account of our ability to consider properties in alternative ways. For, as I have argued elsewhere $(2017,52-$ 54), NM is characterised by an agnostic stance regarding the nature of properties. IDT, however, cannot be agnostic in such a way (on pain of collapsing to NM). Identity theorists should and actually do say something about the nature of properties: they insist that properties themselves (not their conceptions, descriptions or representations) are dispositional and qualitative $e^{23}$. The vital question, however, is whether distinct ways or modes of consideration of a property presupposes distinctions in the world (in particular, a kind of ontological complexity of the property in question). It comes as no surprise that identity theorists answer this question in the negative. If the dispositionality and the categoricality of any property were somehow related to either features or 'parts' of it, then IDT's core identity-claim would obviously be compromised. Heil offers an explanation why some philosophers tend to think that there are distinct features of properties answering to the different modes of their consideration. He proposes that such a line of thinking rests upon a misunderstanding. That is, people who think in that way erroneously consider the case of properties as analogous to the case of concrete objects. Here is Heil:

\footnotetext{
${ }^{23}$ Though relatively obvious, I must stress here that identity theorists do not make the ontological claim that properties are neither dispositional nor qualitative. In fact, they cannot even hold such a view. Properties cannot be identical to their dispositionality and categoricality (as the identity-claim of IDT states) if, by themselves, are neither dispositional nor categorical.
} 
You might object that a capacity for partial consideration presupposes distinctions in the world, and distinctions are a matter of differences in properties. If a property, $\mathrm{P}$, could be considered both as a disposition and as a quality, then, unless we are suffering an illusion, $\mathrm{P}$ must incorporate distinct features answering to these two modes of consideration. Talk of distinguishable features, however, is just an oblique way of indicating distinct properties. Thoughts along these lines maintain an air of plausibility so long as the model for $\mathrm{P}$ is an object. Ordinary objects have multiple properties. Considering the same object in different ways (now as something round, now as something pink) is often a matter of considering distinct properties possessed by the object. In the case before us, however, the focus is on properties, not objects. The model, if you want one, is an ambiguous figure - a Necker cube, for instance - that can be seen now one way, now another. This need not be a matter of attending to different properties of the figure. Rather, we consider the figure as a whole in different ways. (Heil 2003, 119-120)

For Heil, then, in the case of properties, we do not have to appeal to (higher order) properties because what we consider is the whole property in different ways. This is, however, hardly illuminating. Surely, it is inconsistent with IDT to appeal to parts or features of a property in order to (at least partially) explain the ability for considering it in different ways. But even if Heil is right about the suggested difference between objects and properties, his explanation leaves us in the dark. For, what is it about properties in particular that explains the fact that we can consider them in different ways without simultaneously assuming that by doing that we consider distinct (but plausibly inseparable) parts or features of them? In contrast to NM, IDT is expected to give an answer to this question. Leaving it unanswered creates a serious explanatory gap at the very core of IDT. Martin (2008) himself expresses this worry in the following passage: 
Expressing the qualitativity and dispositionality of any real property merely as 'a way of thinking of, mode of prediction concerning, way of regarding or looking at, etc.' suggests that it is merely in the eye (or voice) of the beholder. If those who use such deontologizing expressions intend to claim anthropomorphism, then they should make that ontology fully explicit. If they do not intend to endorse anthropomorphism, however, they should join in the task of saying clearly what in the world the expressions indicate. (Martin 2008, 85)

If what I said above it true, identity theorists have not hitherto achieved the goal 'of saying clearly what in the world the expressions indicate'.

The objection just described is quite general and cannot be met by arguing (as Schroer (2010) and Ingthorsson (2013) do) that the alternative characterisations of one and the same property, irrespective of their content, are (or can be) consistent with each other. Nevertheless, an identity theorist might try to address it by claiming that her ontological account of properties does not aim to explain the epistemic ability to conceive or describe them in different ways. Yet, if the supposed identity between dispositionality and qualitativity has nothing to do with our ability to appeal to distinct dispositional and qualitative characterisations of the same property, then what entitles identity theorists to even speak about qualitativity and dispositionality at the ontological level in the first place? Why not express the claim about the nature of properties in entirely neutral terms? Consider an analogy with the case of Venus. After discovering that this planet is identical to both morning star and evening star, it seems misleading to keep describing it in the old terms and merely add that the different descriptions refer to the same object. Even if the appeal to the qualitative/dispositional ways of speaking is a necessary first step for the clear exposition of their ontological account (in Wittgensteinian terms, a 'ladder' that we can throw away later), why identity theorists keep using a non-neutral vocabulary after at least twenty years since the introduction of IDT? 
Concluding, it is important to stress that the arguments of this section do not aim to show that IDT cannot in principle close the aforementioned explanatory gap. My modest point is that according to the currently available interpretations of IDT's identity-claim, it is eventually a brute ontological fact that a property is able to 'ground' (at least partially) its alternative descriptions/conceptions. That, I submit, brings IDT dangerously close to NM.

To recap: if my arguments in this section are sound, then IDT faces (in addition to the problems identified in Sections 2 and 3) another serious difficulty: there is currently no plausible understanding of its core triple identity-claim that can adequately address DIO. Adding this conclusion to the results of the previous sections seems to make a strong case against IDT (at least in its current form) ${ }^{24}$.

\section{Conclusion}

IDT promises to be a metaphysically interesting middle path between pure powerism and pure categoricalism. In this paper, however, I argued that IDT has not thus far delivered the goods. First, IDT needs a clear positive definition of qualitativity which is both adequate and compatible with the tenets of the theory. Yet, the fact that all the extant proposals fail to provide such a definition strongly suggests that the prospects of providing it are bleak. Second, most of the suggested by IDT-ists conceptions of qualitativity are characterised by a metaphysical 'neutrality' that threatens to trivialise the important metaphysical debate regarding the question whether properties confer by themselves dispositions on their bearers (and so they are inherently modal) or need the 'assistance' of laws of nature to do that. Last but not least, there is the objection concerning the very intelligibility of the core identity-claim of IDT. It is argued that none of the extant alternative understandings of the identity in question can adequately

\footnotetext{
${ }^{24}$ Taylor (2018) argues that IDT and pure powerism are two metaphysical positions that in fact accept the same view of properties. If Taylor's arguments are sound, IDT is surely in trouble because it is supposed to be a middle view between the 'extremes' of pure powerism and pure categoricalism. This paper aims to show that IDT faces serious difficulties even granted its distinctness from the rival theories.
} 
meet the intelligibility objection. Although the conclusions reached here do not prove that IDT cannot address all these problems, they certainly show that identity theorists have a lot of work to do in order to meet the difficulties besetting their theory. At the very least, the challenges that this paper raises may motivate philosophers to explore the prospects of alternative versions of PQV.

\section{Acknowledgements}

I would like to thank the participants of the $6^{\text {th }}$ Panhellenic Philosophy of Science Conference for their helpful comments on an earlier version of this paper. I am also indebted to the anonymous reviewers for their valuable remarks which significantly improve the quality of the paper.

\section{References}

Armstrong, D.M. (1989). A Combinatorial Theory of Possibility. Cambridge: Cambridge University Press.

Armstrong, D.M. (1997). A World of States of Affairs. Cambridge: Cambridge University Press.

Bartels, A. (2016). How to Bite the Bullet of Quidditism-Why Bird's Argument against Categoricalism in Physics fails. In Felline, L., Ledda, A., Paoli, F. and Rossanese, E. (Eds) SILFS Vol.3 New Directions in Logic and the Philosophy of Science, Milton Keynes: College Publ., 87-96.

Bird, A. (2007). Nature's Metaphysics: Laws and Properties. Oxford: Clarendon Press.

Blackburn, S. (1990). Filling in Space. Analysis 50(2), 62-65.

Carruth, A. (2016). Powerful Qualities, Zombies and Inconceivability. The Philosophical Quarterly 66(206), 25-46.

Coates, A. (forthcoming). Making Sense of Powerful Qualities. Synthese https://doi.org/10.1007/s11229-020-02576-5.

Contessa, G. (2015). Only Powers Can Confer Dispositions. The Philosophical Quarterly 259, $160-176$.

Contessa, G. (2019). Powerful Qualities or Pure Powers? Metaphysica 20(1), 5-33.

Cowling, S. (2015). Non-qualitative Properties. Erkenntnis 80(2), 275-301.

Ellis, B. (2001). Scientific Essentialism. New York: Cambridge University Press.

Engelhard, K. (2010). Categories and the Ontology of Powers: A Vindication of the Identity Theory of Properties. In Marmodoro, A. (ed.) The Metaphysics of Powers, New York: Routledge, 41-57.

Giannotti, J. (forthcoming). The Identity Theory of Powers Revised. Erkenntnis https://doi.org/10.1007/s10670-019-00122-5. 
Hawthorne, J. (2001). Causal Structuralism. Nô̂s 35, 361-378.

Heil, J. (2003). From an Ontological Point of View. New York: Oxford University Press.

Heil, J. (2010). Powerful Qualities. In Marmodoro (ed.) The Metaphysics of Powers, Routledge, 58-72.

Heil, J. (2012). The Universe As We Find It. Oxford: Oxford University Press.

Ingthorsson, R.D. (2013). Properties: Qualities, Powers or Both? Dialectica 67(1), 55-80.

Ioannidis, S., Livanios, V. and Psillos, S. (2021). No laws and (thin) powers in, no (governing) laws out. European Journal for Philosophy of Science 11:6.

Jacobs, J. (2011). Powerful Qualities, Not Pure Powers. The Monist 94(1), 81-102.

Jaworski, W. (2016). Structure and the Metaphysics of Mind. Oxford: Oxford University Press.

Livanios, V. (2010). Symmetries, Dispositions and Essences. Philosophical Studies 148(2), 295-305.

Livanios, V. (2017). Science in Metaphysics: Exploring the Metaphysics of Properties and Laws. Cham: Palgrave Macmillan.

Livanios, V. (2018). Do Categorical Properties Confer Dispositions on Their Bearers? Kriterion 32(2), 61-82.

Livanios, V. (2020). Powerful Qualities Beyond Identity Theory. Metaphysica 21(2), 279-295.

Lowe, J. (2006). The Four-Category Ontology: A Metaphysical Foundation for Natural Science. Oxford: Clarendon Press.

Macbride, F. (2020). Truthmakers. The Stanford Encyclopedia of Philosophy (Spring 2020

Edition), E.N. Zalta (ed.), https://plato.stanford.edu/archives/spr2020/entries/truthmakers/>.

Mackie, J.L. (1977). Dispositions, Grounds, and Causes. Synthese 34, 361-370.

Martin, C.B. (1993). Powers for Realists. In Bacon, J., Campbell, K., and Reinhardt, L. (eds) Ontology, Causality and Mind: Essays in Honour of D.M. Armstrong, Cambridge: Cambridge University Press, 175-186.

Martin, C.B. (1996). Properties and Dispositions. In Crane, T. (Ed.) Dispositions: A Debate. London and New York: Routledge, 71-87.

Martin, C.B. (1997). On the Need for Properties: The Road to Pythagoreanism and Back. Synthese 112, 193-231.

Martin, C.B. (2008). The Mind in Nature. Oxford: Clarendon Press.

Martin, C.B and Heil J. (1999). The Ontological Turn. Midwest Studies in Philosophy 23, 3460.

McKitrick, J. (2018). Dispositional Pluralism. Oxford: Oxford University Press.

Mellor, D.H. (2000). The Semantics and Ontology of Dispositions. Mind 109.436, 757-780.

Molnar, G. (2003). Powers: A Study in Metaphysics. New York: Oxford University Press.

Mumford, S. (1998). Dispositions. New York: Oxford U.P.

Mumford, S. (2004). Laws in Nature. London: Routledge.

Oderberg, D. (2009). The Non-identity of the Categorical and the Dispositional. Analysis 69(4), $677-684$.

Schroer, R. (2010) Is There More than One Categorical Property? The Philosophical Quarterly 60(241), 831-850.

Schroer, R. (2013). Can a Single Property Be Both Dispositional and Categorical? The "Partial Consideration Strategy", Partially Considered. Metaphysica 14, 63-77.

Strawson, G. (2008). The Identity of the Categorical and the Dispositional. Analysis 68(4), 271-282.

Taylor, J.H. (2013). In Defence of Powerful Qualities. Metaphysica 14(1), 93-107.

Taylor, H. (2018). Powerful Qualities and Pure Powers. Philosophical Studies 175, 1423-1440.

Tugby, M. (2012). Rescuing Dispositionalism from the Ultimate Problem: Reply to Barker and Smart. Analysis 72(4), 723-731. 